\title{
Arbor
}

\section{Participación pública en política tecnológica: Problemas y perspectivas}

\author{
J. A. López Cerezo, J. A. Méndez Sanz y Oliver Todt
}

Arbor CLIX, 627 (Marzo 1998), 279-308 pp.

Un lugar común en nuestros días es reconocer la gran importancia que tiene hoy el cambio tecnológico. El ritmo creciente de ese cambio, el enorme volumen de recursos públicos que consume y los evidentes riesgos e impactos asociados a la tecnología, han hecho que ésta ocupe hoy un lugar destacado en las agendas políticas, los medios de comunicación y la opinión pública. En efecto, las ultimas décadas han sido testigo de una creciente sensibilización social sobre los problemas relacionados con políticas de innovación tecnológica e intervención ambiental, incluyendo por supuesto tecnologías sociales y organizativas como el sistema de salud o la planificación urbana $^{1}$. No es por tanto una sorpresa que la participación pública en estas politicas constituya hoy día un importante reto para las sociedades democráticas. En la primera parte de este trabajo comentaremos los motivos y condiciones de dicha participación, y revisaremos críticamente las principales iniciativas institucionales que, en tal sentido, han sido ensayadas o propuestas para democratizar el cambio tecnológico. Sobre esta base, en la segunda parte, realizaremos una valoración de la realidad española y discutiremos la posibilidad de adaptar a la misma este tipo de iniciativas.

\section{La base de la participación}

Son varios los factores que subyacen al aumento en la sensibilidad social sobre el cambio tecnológico y la exigencia de respuestas institucionales. Podemos agruparlos en cuatro grandes bloques ${ }^{2}$ : 


\section{Nueva imagen de la ciencia y la tecnología}

Cabe señalar, para empezar, una cierta ambivalencia en la percepción popular: se hacen evidentes tanto los efectos positivos como las consecuencias negativas de la ciencia y la tecnología actual. En las últimas décadas se extiende la opinión de que la tecnología, a pesar de sus logros, ha fracasado en resolver muchos problemas sociales y ha contribuido a crear otros nuevos.

En este sentido, aparecen nuevas preocupaciones sociales en torno a la ciencia-tecnología:

- Riesgos (energía nuclear, residuos tóxicos, fertilizantes químicos, etc.).

- Implicaciones éticas (experimentación con embriones humanos, uso comercial de información genética, madres de alquiler, etc.).

- Uso discutido de descubrimientos científicos o innovaciones tecnológicas (ADN recombinante, diferencias sexuales, bancos de datos, etc.).

- Equitatividad en la distribución de recursos y de costes sociales y ambientales.

No es entonces sorprendente el desarrollo de movimientos de resistencia social frente a diversas iniciativas en política tecnológica 0 ambiental (pruebas nucleares, control informático, aviación comercial supersónica, programa espacial, etc.).

Otro factor relacionado es la disminución del prestigio social de los científicos de acuerdo con las encuestas de opinión, junto con el prestigio general de las instituciones. Los medios de comunicación han contribuido a transmitir la imagen de la ciencia como un cuerpo de conocimiento falible $y$, en ocasiones, fraudulento.

Adicionalmente, los nuevos estudios sociales sobre ciencia y tecnología, que comienzan a difundirse en el ámbito educativo general y de periodismo científico en los años 80 , están contribuyendo a desmitificar la ciencia y problematizar la tecnología al mostrar estas actividades como procesos sociales que dependen en parte de valores e intereses «externos» ${ }^{3}$.

\section{Nuevo papel de los expertos}

El conocimiento especializado adquiere una importancia política cada vez mayor en los distintos niveles de la administración. Se produce una institucionalización del asesoramiento experto, junto con 


\section{Participación pública en política tecnológica}

un cambio en la naturaleza del ejercicio del poder y los medios de control social ${ }^{4}$.

Sin embargo, no está clara la naturaleza del papel político de los expertos:

- Para algunos, estamos hablando de simple tecnocracia, de una nueva fuente de poder no democrático con influencia sobre políticos y sobre el público en general. Se trata de la llamada teoría de los expertos como tecnócratas.

- Para otros, los expertos son mantenidos por el poder político y disfrutan de poco o ningún poder real; son simplemente utilizados como tapadera para legitimar decisiones tomadas por otros motivos. Se trata de la llamada teoría de losi expertos como mandarines.

En cualquier caso tiene lugar un socavamiento de los valores democráticos.

\section{Nuevo concepto de participación sociopolitica}

Se produce un creciente interés ciudadano en participar más en la toma de decisiones en políticas públicas; interés paralelo a la desconfianza en la metodología opaca tradicionalmente utilizada en la evaluación y gestión del cambio tecnológico. Aunque no se trata, en todo caso, de impugnar el sistema democrático sino de profundizar en él: el ciudadano es cada vez más consciente de que su soberanía no se agota en la elección periódica de representantes políticos que rinden cuentas cada determinado tiempo.

Por otro lado, la administración pública en los países industrializados es cada vez más consciente de los problemas y limitaciones producidos por una orientación tecnocrática de la política tecnológica o ambiental.

En este sentido, otro factor importante son los nuevos estudios cualitativos y cuantitativos sobre comprensión pública de la ciencia, unos estudios que han socavado el mito de la relación entre ignorancia de la ciencia-tecnología y oposición a la misma ${ }^{5}$.

\section{Nueva imagen del ser humano como miembro del medio biosocial}

Se extiende el sentimiento de que la biosfera es un sistema frágil y único, algo cuya continuidad amenazamos como especie al actuar sobre él de un modo abusivo por medio de la tecnología moderna. 
Paralelamente, se tiende a reconocer que depende de nosotros evitar que la obtención de recursos vitales acabe con su fuente. Se impone así un creciente sentimiento de responsabilidad con respecto a generaciones futuras y segmentos sociales desfavorecidos.

La imagen de un mundo único se ve reforzada continuamente por la tendencia político-económica a resolver los problemas actuales mediante la coordinación de proyectos a escala mundial (CFCs y la capa de ozono, recursos pesqueros, selva tropical, etc.). La continua difusión por los medios de comunicación de la imagen planetaria de la tierra, y el reconocimiento de la potencialidad de la tecnología moderna, hace que sea imposible reducir el alcance de las acciones tecnológicas y ambientales a su impacto local: la producción de riesgos y daños rebasa hoy los tradicionales límites temporales, espaciales y de clase social ${ }^{6}$.

Paralelamente, se produce una revalorización de los nichos singulares y la diversidad. Su preservación es crecientemente enfatizada en distintas políticas de desarrollo asociadas a la innovación tecnológica o la intervención ambiental.

Todos estos factores se encuentran en la base de la reciente sensibilización pública respecto al cambio tecnológico. Como resultado, las relaciones entre tecnología y sociedad se encuentran hoy en un proceso de renegociación política ${ }^{7}$.

\section{El horizonte de la participación}

Siguiendo a Daniel Fiorino (1990), podemos resumir los motivos para la participación pública en tres argumentos (que son los que están en juego en dicho proceso de negociación política):

- La participación es la mejor garantía para evitar la resistencia social y la desconfianza hacia las instituciones (argumento instrumental).

- La tecnocracia es incompatible con los valores democráticos (argumento normativo).

- Los juicios de los no expertos son tan razonables como los de los expertos (argumento substantivo) ${ }^{8}$.

El núcleo de la cuestión no es tanto imponer límites a priori al desarrollo de la ciencia y la tecnología, sino renegociar quién debería decidir objetivos políticos en ciencia y tecnología y quién debería supervisar su cumplimiento. Los lemas de esta renegociación son bien 


\section{Participación pública en política tecnológica}

conocidos: "participación popular», "responsabilidad social de los expertos", "ciencia para el pueblo", "tecnología en democracia", etc.

De este modo, superar los obstáculos que dificultan la participación pública en política tecnológica es algo reconocido, cada vez con más fuerza, como una de las condiciones necesarias para una gestión social y ambientalmente adecuada del cambio tecnológico. La tradicional rendición de cuentas cada cuatro años por parte de gobiernos y parlamentos en sociedades democráticas, ha demostrado ser, desde el punto de vista de muchos colectivos sociales, una forma indirecta de control social demasiado endeble ante un cambio científico-tecnológico cada vez más vertiginoso y que plantea problemas más y más apremiantes ${ }^{9}$.

No obstante, la identificación de actores sociales y la coordinación de sus intereses en la participación pública es una tarea que está lejos de ser sencilla debido a la disparidad de puntos de vista, grado de información, concienciación y poder de cada uno. Por ello, debemos ahora detenernos brevemente a considerar quién puede participar, y cómo, en una política tecnológica democrática.

\section{Las posibilidades de la participación}

¿Qué ciudadanos o grupos sociales, qué "públicos", en suma, están involucrados o pueden involucrarse en la gestión pública de la ciencia y la tecnología, sumándose así a los que controlan este proceso actualmente? ${ }^{10}$.

\section{Tipos de ciudadano}

* Personas directamente afectadas por la innovación tecnológica o la intervención ambiental que no pueden evitar el riesgo o el impacto directo (económico, ambiental, ...), por ejemplo los vecinos de una central nuclear. Se trata del público más obvio y del que proceden la mayoría de reclamaciones de participación. Se pone normalmente en cuestión la justicia de la distribución prevista de costes y beneficios, o la consideración de riesgos físicos a través de una metodología de coste/beneficio.

* Público involucrado. Se trata de receptores directos de servicios profesionales o instalaciones tecnológicas como los pacientes médicos de los sistemas de salud. Se ven potencialmente afectados de un modo directo (es decir, son personas directamente afectadas en potencia). 
* Consumidores de los productos de la ciencia-tecnología. Se trata de un público más vagamente definido que suele protestar contra las regulaciones o diversos usos de la tecnología. Por ejemplo, sobre el uso de ciertos fertilizantes en agricultura, hormonas en ganadería, aditivos químicos en alimentación, etc.

* Público interesado. Se trata de personas concienciadas o sensibilizadas particularmente sobre los problemas tecnológicos o ambientales por sus principios morales o ideológicos. Suelen pertenecer o ser simpatizantes de grupos ecologistas $u$ organizaciones no gubernamentales diversas.

* Comunidad científica e ingenieril. La politización de estas comunidades durante los años 60 ha producido la protesta organizada de muchos científicos e ingenieros contra la carrera armamentista 0 , más recientemente, contra la energía nuclear o la investigación biomédica ${ }^{11}$.

\section{Tipos de organización}

* Grupos de ciudadanos. Son coaliciones temporales, a veces organizadas espontáneamente, que se forman para protestar contra decisiones, proyectos o políticas específicas. Pueden actuar a nivel local (por ejemplo, asociaciones de vecinos coordinados en acciones comunitarias) o a nivel nacional (caso de la protesta contra General Electric organizada por Infact en EE.UU.). No siempre se disuelven tras la resolución de la disputa, a veces permanece un núcleo de activistas con poder para nuevas movilizaciones.

* Organizaciones no gubernamentales (ONGs), de carácter regional (Asociación Asturiana de Amigos de la Naturaleza), nacional (AEDENAT en España) o internacional (Greenpeace). Tratan en general de promover la rendición de cuentas mediante el escrutinio público. Además de movimientos ecologistas, con frecuencia se ven involucrados en las disputas otros tipos de grupo de interés: asociaciones de consumidores, sindicatos, partidos políticos, etc. ${ }^{12}$.

* Asociaciones de científicos. Por ejemplo "Science for the People», "British Society for the Social Responsibility in Science", "Coalition for Responsible Genetic Research", "Center for Concerned Engineers", etc. Se trata de asociaciones de científicos de izquierda o con un cierto compromiso social ${ }^{13}$. Suelen brindar un conocimiento técnico alternativo para apoyar a grupos de ciudadanos y ONGs.

El problema general de quién debe o puede participar en controversias relacionadas con la tecnología o el medio ambiente es obviamente 


\section{Participación pública en política tecnológica}

una cuestión de la mayor importancia política. Por ejemplo, en la reciente polémica acerca de la ampliación del Parque Nacional de la Montaña de Covadonga hasta el Parque Nacional de los Picos de Europa, uno de las cuestiones más debatidas era la determinación del colectivo o colectivos cuya opinión debía ser considerada y, en su caso, priorizada. ¿Qué opinión es la importante? ¿Sólo la de los habitantes locales directamente afectados, que quieren continuar con su tradicional aprovechamiento de la tierra y seguir con las batidas de lobos? ¿También la de sus representantes en las administraciones local, autonómica y nacional de las tres comunidades autónomas afectadas, aunque no terminen de ponerse de acuerdo? ¿Deberían acaso participar los asturianos, cántabros y castellano-leoneses en su conjunto, aunque vivan en Gijón, Torrelavega o Ponferrada? ¿O quizá también el ciudadano concienciado de Valencia, que visita la zona en Semana Santa y desearía la máxima protección? ¿Acaso es competencia de Bruselas? Como podemos ver no son preguntas fáciles de responder.

\section{Las condiciones de la participación democrática}

¿Qué condiciones, entonces, ha de cumplir la gestión pública en política tecnológica para poder ser considerada suficiente desde un punto de vista democrático? De forma realista (es decir, no planteando un programa de máximos), las siguientes condiciones son habitualmente señaladas en la literatura sobre participación pública ${ }^{14}$ :

* Carácter representativo: debe producirse una amplia participación en el proceso de toma de decisiones. En principio, cuanto mayor sea el número de grupos o el porcentaje de individuos involucrados, y más diversa sea la extracción de éstos, más democrático puede considerarse el mecanismo participativo en cuestión.

* Carácter directo e igualitario: debe permitir la participación directa de los ciudadanos no expertos (a título individual o agrupados) en pie de igualdad con los expertos y las autoridades gubernamentales. Ello implica, en principio, transmisión de toda la información, disponibilidad de medios, tiempo para el debate, no intimidación, igualdad de trato y transparencia en el proceso.

* Carácter efectivo: debe traducirse en un influjo real sobre las decisiones adoptadas en todas las fases del proceso, desde la discusión básica hasta la ejecución de lo acordado. Para ello es necesario que se produzca una delegación de la autoridad o un acceso efectivo a aquellos que la detentan. 
* Carácter activo: debe permitir al público participante involucrarse activamente en la definición de los problemas y el debate de sus parámetros principales, y no sólo considerar reactivamente su opinión en el terreno de las soluciones (con lo que se limitaría a optar entre un ramillete de posibilidades determinadas de antemano por expertos o autoridades). Así, el proceso de participación, más que un mecanismo de toma de decisiones compartidas, se convierte en un proceso de aprendizaje social ${ }^{15}$, en un modo de participación integral en el que no hay puertas cerradas de antemano.

Estas condiciones nos servirán como criterio para evaluar las formas de participación que consideraremos a continuación ${ }^{16}$.

\section{Articulación institucional de la participación}

Veamos ahora las principales posibilidades de participación pública que han sido propuestas o realizadas en diversos países de nuestro entorno cultural.

En España, la participación pública en el ámbito legislativo-ejecutivo está en gran parte limitada a la elección de representantes políticos y éstos, en general, actúan supeditados a las políticas generales de sus partidos. Aunque existen algunas posibilidades de influencia más directa, sólo en pocas ocasiones estas vías de participación ciudadana han tenido una influencia decisiva en este país (véase la discusión del caso español más adelante).

En el ámbito judicial español, igualmente, la capacidad ciudadana de influir en política tecnológica o ambiental mediante denuncias es también reducida, debido a la insuficiencia de la legislación al respecto. Aunque los avances recientes en esta materia (véase más adelante) indican posibles cambios, sigue siendo imprescindible considerar las iniciativas llevadas a cabo en otros países occidentales, más dinámicos en este terreno. Por ejemplo, en Estados Unidos, Australia, Alemania, Suecia o los Países Bajos ${ }^{17}$, se han ensayado diversos mecanismos que posibilitan, en diferente medida, la participación ciudadana.

Por un lado, en el ámbito administrativo:

* Las audiencias públicas. Corresponden a un tipo genérico de mecanismo participativo. Son habitualmente foros abiertos y poco estructurados donde, a partir de un programa previamente determinado por los representantes de la administración, se invita al público a escuchar las propuestas gubernamentales y comentarlas. El público 
participante normalmente procede de los miembros más educados y políticamente activos de la comunidad, así como de los grupos de interés implicados. Como señala Fiorino (1990: 230), "[las audiencias] dan al menos la apariencia de implicación individual y comunitaria, legitiman decisiones ya tomadas, alertan a la administración de obstáculos potenciales, satisfacen requisitos legales o administrativos, y socavan la oposición". Las audiencias públicas, con frecuencia, son sólo una parte de programas de participación más amplios ${ }^{18}$.

* La gestión negociada. Este mecanismo ha sido introducido en Estados Unidos y otros países como complemento del procedimiento tradicional de información-comentario. Ante un asunto determinado (por ejemplo la discusión sobre un reglamento específico), se constituye un comité negociador compuesto por representantes de la administración y grupos de interés implicados, por ejemplo la industria, asociaciones profesionales y organizaciones ecologistas. Los participantes tienen acceso a la información relevante, así como la oportunidad de persuadir a otros y alinearlos con su posición. Este tipo de comité goza de una considerable autonomía sobre procedimientos a seguir, manejo de recursos, definición de problemas, determinación de plazos, etc. Los representantes gubernamentales se comprometen (en la medida que estén autorizados) a asumir públicamente como propio el posible consenso alcanzado por el conjunto de las partes ${ }^{19}$.

* Los paneles de ciudadanos. Este tipo de mecanismo está basado en el modelo del jurado, aunque aplicado a temas científico-tecnológicos y ambientales. Bajo este epígrafe pueden agruparse tanto modelos teóricos como ya realizados ${ }^{20}$, así como paneles con carácter decisorio o meramente consultivos ${ }^{21}$. En general, la idea que los inspira es que ciudadanos corrientes (elegidos por sorteo o por muestreo aleatorio estratificado) se reúnan a considerar un asunto en el que no son expertos. Tras haber recibido información de peritos y autoridades, los ciudadanos han de discutir alternativas y emitir recomendaciones a los organismos oficiales ${ }^{22}$. Los expertos y los representantes de grupos de interés pueden también tomar parte en el panel. Estos paneles, al contrario que las más comunes audiencias públicas, permiten una búsqueda activa de evidencia, interrogar a expertos y una exploración más profunda de los problemas abordados.

* Las encuestas de opinión sobre diversos asuntos relacionados con la innovación tecnológica o la intervención ambiental. Su propósito es proporcionar un testimonio de la percepción pública sobre un asunto determinado, de modo que pueda ser tenida en cuenta por el legislativo o el ejecutivo. Las audiencias públicas pueden completar este mecanismo 
proporcionando una imagen más fina de las actitudes públicas y de su intensidad sobre una determinada cuestión ${ }^{23}$.

Por otra parte, en el ámbito legislativo y judicial, más familiares para nosotros son:

* La realización de referenda y la litigación, que se han convertido en muchos países occidentales en el principal procedimiento que tienen los ciudadanos para restringir y dirigir el cambio tecnológico. Son mecanismos prototípicamente democráticos puesto que todo ciudadano puede participar y el resultado es vinculante para la administración ${ }^{24}$.

\section{Discusión de los mecanismos institucionales}

En general, y a pesar de sus aspectos positivos, todos estos procedimientos presentan inconvenientes o rasgos mejorables ${ }^{25}$. Para empezar, en las audiencias públicas el ciudadano participa sobre todo reactivamente, es decir, no contribuye a definir el problema sino que lo encuentra ya formulado en un sentido determinado por las autoridades o los expertos. La solemnidad del ambiente puede intimidarle $y$, en todo caso, es la autoridad quien decide y encauza las discusiones, resultando difícil plantear cuestiones $u$ objeciones fuera de una agenda previamente establecida. Además, no hay autoridad compartida y existe una profunda asimetría de medios con respecto a expertos y representantes de la administración.

En los paneles consultivos, el ciudadano común también está en desventaja frente a los expertos y lo que se suele discutir es el cómo y no el qué, que ya está decidido. No obstante, en estos paneles se produce una mayor igualdad en la participación ciudadana (con respecto a expertos y políticos) debido a un mayor grado de delegación de autoridad. Por otra parte, debe admitirse que se trata de procedimientos caros y largos (debido a que los no expertos han de asimilar una gran cantidad de información), y, a diferencia de una encuesta o un referéndum, estos mecanismos tienen una representatividad limitada.

El litigio entraña también multitud de problemas sobre procedimientos jurídicos, consejo especializado, largos plazos temporales, etc., aunque tiene la ventaja de permitir la participación de personas o grupos no directamente afectados por el asunto en cuestión. Los referenda presentan muchas debilidades (respuesta reactiva a cuestiones cerradas, limitación de alternativas, ausencia de un debate articulado, etc.), al igual que las encuestas. No obstante, los referenda, a diferencia de las encuestas, suelen propiciar la discusión pública sobre los asuntos 


\section{Participación pública en política tecnológica}

a considerar y tienen un mayor carácter directo y efectivo puesto que los ciudadanos participan de un modo igualitario y con efectos habitualmente decisorios.

Aunque la gestión negociada es, de momento, un procedimiento más bien complementario, y su legitimidad en asuntos que conciernen a valores sociales o elecciones fundamentales es limitada (debido a la forma de elegir sus miembros) ${ }^{26}$, este tipo de mecanismo presenta rasgos democráticamente prometedores y susceptibles de desarrollo (i.e., mayor representatividad, posibilidad de participación activa, directa e igualitaria, y posibilidad de influir en la toma de decisiones). Una importante debilidad de la gestión negociada, sin embargo, es que, al no dar entrada a la participación ciudadana directa, no favorece el aprendizaje social y la discusión pública del modo en que pueden hacerlo otros mecanismos.

Todos estos procedimientos presentan, en suma, puntos débiles y puntos fuertes, dependiendo del criterio de participación democrática considerado y, en última instancia, de la teoría de la democracia presupuesta ${ }^{27}$. En casos prácticos, es conveniente adecuar el mecanismo de participación a las características concretas que se presenten en cada situación. Por ejemplo, ante problemas fuertemente ideologizados no es conveniente un procedimiento de participación que involucre la interacción cara-a-cara, puesto que tiende a radicalizar las posturas; mientras que ante decisiones concernientes a localización de recursos tal forma de interacción es viable y positiva ${ }^{28}$. Algunas cuestiones relativas a, por ejemplo, el riesgo o impacto social de nuevas tecnologías pueden requerir un mecanismo que favorezca una amplia discusión social (como un referéndum); otras cuestiones, donde estén en juego la distribución de recursos o riesgos, harían aconsejable un procedimiento que no descontextualice las opiniones e intereses de los diferentes actores (excluyendo así, por ejemplo, las encuestas). En otras ocasiones, por ejemplo ante problemas ambientales de carácter local, puede ser conveniente favorecer el aprendizaje social a través de mecanismos de participación directa (como paneles consultivos); mientras que en casos donde el problema afecte a mucha gente distribuida en diversos segmentos sociales, parece preferible la representación a través de grupos de interés (como en la gestión negociada).

\section{Evaluación de las posibilidades de participación}

Con todo, el grado de la participación pública puede ser tentativamente evaluado en función del modo en que se articule institucio- 
nalmente $\mathrm{y}$, en última instancia, dependiendo de la sensibilidad democrática de las políticas públicas en la gestión del cambio tecnológico. Sobre la base de la discusión anterior, es posible proponer una clasificación de los argumentos y mecanismos participativos que puede ser de utilidad para evaluar las iniciativas institucionales y detectar las realidades nacionales.

participación?

\begin{tabular}{|c|c|c|c|c|}
\hline (i) por qué? & (ii) de quién? & (iii) para qué? & (iv) cómo? & (v) riesgos \\
\hline $\begin{array}{l}\text { sumento } \\
\text { trumental }\end{array}$ & $\begin{array}{l}\text { percepción } \\
\text { pública }\end{array}$ & $\begin{array}{l}\text { evitar resistencia } \\
\text { social }\end{array}$ & $\begin{array}{l}\text { mecanismos } \\
\text { de sondeo }\end{array}$ & manipulación \\
\hline $\begin{array}{l}\text { zumento } \\
\text { mativo }\end{array}$ & $\begin{array}{l}\text { opinión } \\
\text { pública }\end{array}$ & $\begin{array}{l}\text { legitimidad } \\
\text { democrática }\end{array}$ & $\begin{array}{l}\text { mecanismos } \\
\text { reactivos }\end{array}$ & desconfianza \\
\hline $\begin{array}{l}\text { umento } \\
\text { stantivo }\end{array}$ & $\begin{array}{l}\text { conocimiento } \\
\text { público }\end{array}$ & co-decisión & $\begin{array}{l}\text { mecanismos } \\
\text { activos }\end{array}$ & $\begin{array}{l}\text { inherentes } \\
\text { a la gestión }\end{array}$ \\
\hline
\end{tabular}

Tenemos así una propuesta de clasificación evaluativa de las distintas concepciones de la participación. La primera línea resume una concepción que se basa únicamente en el argumento instrumental de Fiorino (a saber, la participación previene la resistencia social). En esta concepción, la participación pública se reduce a percepción pública, de forma que el objetivo de una política democrática se reduciría a preservar una percepción pública positiva o cambiar una percepción negativa de forma que se prevenga la ineficacia derivada de una posible resistencia social. Apropiadamente llevados a cabo, los mecanismos de sondeo como encuestas de opinión o audiencias públicas son concebidos aquí como mecanismos suficientes para la participación. Como ilustraremos más adelante, éste parece ser el umbral habitual de la participación pública en España: el de una gestión defensiva.

En la segunda línea del diagrama, donde encontramos una concepción de la participación basada en el argumento normativo de Fiorino (es decir, excluir la participación es incompatible con los valores democráticos), la participación tiende a ser identificada con opinión pública: los actores sociales deben tener la posibilidad de expresar su opinión a fin de conferir legitimidad al proceso de toma de decisiones. No obstante, en esta concepción, la participación pública es fácilmente vista como una clase de necesaria interferencia externa. Es normal entonces que tiendan a promoverse mecanismos reactivos del tipo comisión asesora, audiencia pública o variedades no-decisorias de la participación. Un claro riesgo de esta concepción es, entre los ciudadanos, la formación de desconfianza hacia las instituciones (por la escasa 
posibilidad en este tipo de participación de influir sustancialmente en la toma de decisiones), y, entre los expertos, la percepción de dificultades para una gestión eficiente.

Por último, en la tercera línea encontramos una concepción de la participación basada en el argumento substantivo (es decir, el juicio de los no expertos es tan sensato y valioso como el de los expertos). Esta concepción expande las posibilidades participativas hasta incluir el conocimiento popular local, entendido como una fuente autorizada de información y perspectivas. Se presupone aquí que los ciudadanos afectados y el público en general tiene o puede adquirir la información que los capacite para formar una juicio fiable sobre problemas relacionados con la tecnología ${ }^{29}$. Es natural entonces que, en esta concepción, se promuevan mecanismos más activos, efectivos e igualitarios, por ejemplo los paneles de ciudadanos o la gestión negociada con poder decisorio.

Por la importancia de este tercer argumento, es interesante ver un sencillo ejemplo del modo en que los afectados pueden proporcionar un conocimiento útil y relevante. Cuando los agricultores británicos comenzaron a protestar a finales de los años 60 por los efectos de diversos herbicidas sobre la salud, especialmente el 2,4,5-T o agente naranja (usado también por entonces como defoliante en la guerra de Vietnam), el gobierno de ese país pidió una investigación a un comisión de expertos (el Pesticides Advisory Committee), compuesta fundamentalmente por toxicólogos. En su trabajo durante los años 70, la comisión se centró en la literatura sobre toxicología de los agentes químicos en cuestión. La conclusión inequícova fue que no había riesgo alguno para la salud humana. La respuesta de los agricultores, constituidos en grupo de interés (el National Union of Agricultural and Allied Workers - Sindicato Nacional de Trabajadores del Campo), fue enviar al gobierno un informe aún más grueso con casos de daño médico; un informe que la comisión de expertos se limitó a desestimar como algo anecdótico, como opinión acientífica y no sistemática.

Los agricultores, sin embargo, continuaron con la presión pública y, nuevamente, la comisión gubernamental afirmó que los herbicidas no causaban daño. Pero, esta vez, los expertos se vieron obligados a introducir un pequeño aunque importantísimo matiz: los herbicidas no causaban daño de acuerdo con la literatura científica, es decir, siempre que fuesen utilizados de un modo correcto. Sobre su modo real de utilización, los agricultores, que no habían sido escuchados, eran los verdaderos expertos. Sabían que las condiciones correctas de utilización eran pura fantasía científica. Las instrucciones de uso se 


\section{José A. Cerezo, José A. Méndez Sanz y Oliver Todt}

ignoraban o perdían con frecuencia, el equipo correcto de aspersión era muchas veces inasequible, el traje protector era inadecuado, y las condiciones atmosféricas eran habitualmente ignoradas bajo la presión de terminar el trabajo ${ }^{30}$.

\section{El caso español}

En España, como hemos mencionado, la articulación institucional de la participación tiende a ser concebida a través del argumento instrumental y limitarse a mecanismos indirectos y reactivos. Esto suele traducirse en un tipo de gestión donde se infravaloran las posibilidades substantivas de la participación y de desarrollo de las estructuras democráticas. Para concluir, y sobre la base de la discusión anterior, vamos a realizar un análisis valorativo de las causas de esta situación al hilo del examen de varios casos concretos, finalizando con una breve reflexión sobre las líneas de acción que quizás puedan contribuir a corregirla.

Debemos, previamente, recordar que España es un país sin una arraigada tradición participativa debido a (entre otras cosas) una reciente dictadura y a largos períodos de gobierno autoritario. Esto, naturalmente, ha tenido una influencia en el ámbito de la ciudadanía y de la administración pública. La sensibilidad pública por estos temas es, además, muy reciente.

\section{Problemas}

En principio, hay que intentar comprender dos fenómenos que se dan en España: por un lado, la reticencia de la administración pública respecto a permitir y fomentar vías de participación, y, por otro, una cierta inhibición de los ciudadanos a utilizar los (ciertamente limitados) mecanismos de participación que existen. Es básicamente la combinación de estos dos factores la que, en nuestra opinión, conduce a la situación comentada.

Reticencia de la administración pública

(a) Gestión defensiva (respecto a los grupos organizados de interés y la opinión pública). Una gestión democrática es entendida aquí a 
través de la concepción instrumental de la participación, es decir, como una gestión que se limita a tener en cuenta la percepción pública y trata de evitar la resistencia social. El significado de una gestión "socialmente sensible" se agota así en la detección de actitudes públicas y posibles fuentes de inestabilidad social con el fin de adaptar en consecuencia las políticas públicas. No es, por tanto, una sorpresa que la negociación tienda a ser concebida como una debilidad o una interferencia más bien que como una ventaja o una necesidad conveniente. Lo mismo es cierto para otros conceptos de participación que implicarían que los ciudadanos se involucren activamente en la administración.

El mejor ejemplo lo presenta la legislación relacionada con el medio ambiente y la regulación de la tecnología, así como su aplicación en la práctica. Dos casos recientes: la Ley sobre el Derecho de Acceso a la Información en Materia de Medio Ambiente y la Ley de Biotecnología. La primera de ellas, conocida como Ley de Información Ambiental ${ }^{31}$, estipula el derecho de todos los ciudadanos a recibir la información manejada por la administración sobre medio ambiente; pero en la práctica esta posibilidad, que sería uno de los requisitos para una mejor información e implicación de los ciudadanos, queda muy limitada. La ley incluye la posibilidad legal de denegación de una solicitud por vía de silencio administrativo, esto es, sin exposición explícita de motivaciones para la denegación. Hasta 1997, desde la entrada en vigor de la ley, la administración se ha acogido a esta posibilidad en el $80 \%$ de los casos. La ley también permite a la administración fijar tasas para el suministro de la información solicitada, que en muchos casos - por su alto importe- han sido calificadas de disuasorias por los ecologistas ${ }^{32}$.

En el caso de la Ley de Biotecnología ${ }^{33}$, ésta otorga a la administración la posibilidad de información pública e incluso la de realizar consultas públicas en el proceso administrativo de autorización de investigaciones con organismos modificados genéticamente (OMG). Sin embargo, excepto cuando se trata de operaciones definidas como de "alto riesgo", la administración tiene completa potestad para iniciar, o no, estos trámites. Habitualmente se suministra la lista de ensayos de campo con OMGs a quien la pide, pero tal lista no contiene más que noticias elementales, no suministrándose datos detallados de, por ejemplo, las evaluaciones de riesgo o de la información en general que se maneja dentro del proceso de autorización. La política de la administración en este caso ha sido responder a peticiones hechas por otras personas, pero no promover activamente la diseminación del tipo de información mencionada. La posibiiidad de participación de 
otros actores sociales, permitida por la ley, no se ha realizado hasta el momento, aunque en principio el Ministerio de Medio Ambiente está dispuesto a ello ${ }^{34}$. Igual que en el caso de la diseminación de información, no se han tomado hasta el momento medidas activas para preparar esta participación, ni desde el Ministerio ni desde los organismos implicados en la regulación, como por ejemplo la Comisión Nacional de Bioseguridad (también hay que tener en cuenta aquí la provisionalidad del funcionamiento de la ley).

Estos ejemplos, como otros del ámbito del urbanismo ${ }^{35}$, muestran que existen posibilidades de participación pero que en la práctica la administración utiliza las posibilidades permitidas por la legislación más bien para limitar esta participación que para fomentarla activamente. En los casos mencionados, el desarrollo de las posibilidades legales de información y participación se interpreta como algo que el ciudadano tiene que pedir a la administración, no como algo que la administración desarrolla de motu propio. El ciudadano, por tanto, se ve obligado a tomar la iniciativa para informarse sobre sus derechos si quiere verlos reconocidos de hecho.

Otro dato a tener en cuenta es que, en la mayoría de los casos, son las exigencias de la legislación europea las que impulsan la ampliación de las posibilidades legales de información y participación, y no la iniciativa propia del legislador español (así lo muestra, por ejemplo, el caso de la evaluación de impacto ambiental) ${ }^{36}$.

La legislación, en teoría, va ampliando poco a poco las posibilidades de participación de los ciudadanos. También las posibilidades jurídicas al alcance de los ciudadanos están mejorando, como lo muestran los recientes desarrollos en materia de medio ambiente: el reconocimiento de delito ecológico en el nuevo código penal o la preparación de una ley de responsabilidad civil ambiental por el Ministerio de Medio Ambiente. Sin embargo, una característica general de la legislación es que sigue dando a la administración un amplio margen de discrecionalidad para iniciar o no mecanismos de participación y que esa participación se da en forma reactiva, es decir, sólo en las fases avanzadas del proceso administrativo, a través de audiencias o informes, pero casi nunca al principio de una acción administrativa ${ }^{37}$.

En cuanto a la práctica de la participación, existen algunas experiencias como, por ejemplo, el Consejo Asesor de Medio Ambiente (CAMA) del anterior Ministerio de Obras Públicas, Transporte y Medio Ambiente. El CAMA, instaurado en el 1994 para asesorar al Ministerio en cuestiones ambientales, contaba con una amplia representación de la sociedad civil, incluyendo sectores ecologistas, de consumidores y 
de otras ONGs. Pero las expectativas de muchas ONGs no se cumplieron, y muchas de ellas se retiraron del Consejo después de un tiempo (tras discusiones sobre casos conflictivos como el embalse de Itoiz o la autovía N-III). El argumento que dieron fue que mientras en el Consejo se podía discutir sobre los proyectos y la gestión del Ministerio de manera bastante fructífera, en la práctica política las resoluciones acordadas en el CAMA no tenían influencia sobre las decisiones del Ministerio. Incluso se llegó a reprochar a la administración haber creado el CAMA para maniatar a las ONGs que, con su participación en el Consejo, perdían sus fuerzas en discusiones sin incidencia sobre la toma de decisiones ${ }^{38}$.

Como conclusión, se puede decir que la administración interpreta las posibilidades legislativas de manera muy restrictiva, lo que ya ha dado lugar a acciones jurídicas contra ella: así pasó con la ya mencionada Ley de Información Ambiental, cuando un grupo de personas no aceptó que su solicitud de información fuera objeto de una negativa no razonada ${ }^{39}$.

(b) Infravaloración de las posibilidades substantivas de la participación. Este punto está relacionado con la falta de experiencias de participación, así como con la sobrevaloración del conocimiento especializado (sobre la base de una visión reductiva de la naturaleza de los problemas sociales). Es lo que ha ocurrido, por ejemplo, con la gestión pública del llamado "problema del eucalipto" en el norte de España, y, particularmente, en Asturias. Desde que estalla este problema en política forestal en la segunda mitad de los años 80 , la administración pública trata de presentarlo como una cuestión fundamentalmente técnica, a saber, como la cuestión de maximizar los beneficios económicos globales de las plantaciones forestales tratando de minimizar los perjuicios ambientales. La acción política se ampara entonces en disposiciones y decretos legales que son interpretados e implementados por expertos al servicio de la administración autonómica, sin trazo alguno de participación pública o implicación de ningún grupo de interés (véase más adelante). Un problema social, que había generado ya una percepción pública negativa a principios de los años 90, se transforma y trata de ser resuelto como una cuestión meramente técnica. Es una tecnificación que ha omitido información local y factores difícilmente cuantificables, conocidos particularmente por la población afectada y relacionados con la tradición cultural, las prácticas económicas en el ámbito rural, el impacto del eucalipto en cultivos colindantes, las especies protegidas presentes en áreas de repoblación, etc. El resultado de esta gestión tecnocrática ha 
sido, entre otras cosas, la persistencia de una percepción negativa e incluso de resistencia social (llegando, en algunos casos, al enfrentamiento con la Guardia Civil) ${ }^{40}$.

En casos como éste podría llegar a decirse que se ha hecho un uso político de la ciencia (de acuerdo quizá con la "teoría del experto como mandarín»), un uso que utiliza la ciencia, y la sobrevaloración del conocimiento especializado por parte de la población, para legitimar una acción política opaca que sólo atiende a los beneficios a corto plazo de la innovación tecnológica o la intervención ambiental.

Reticencias de los ciudadanos

(c) Inhibición pública. Los ciudadanos españoles, en general, tienen un grado de optimismo científico-tecnológico mayor que los demás ciudadanos de la Unión Europea, lo que parece relacionado con el rápido desarrollo que el país ha experimentado en las últimas décadas y la menor importancia que se ha dado a los problemas tecnológicos y ambientales en la percepción pública ${ }^{41}$. Las encuestas también muestran que los españoles tienen una consideración relativamente alta del conocimiento especializado (lo cual contrasta con su baja cultura científico-tecnológica con respecto al conjunto de países comunitarios) ${ }^{42}$. Aquí radica uno de los factores que influyen en el menor activismo en España en cuestiones relacionadas con efectos negativos del cambio tecnológico.

Debemos además tener en cuenta una cierta desconfianza institucional que es consecuencia de la larga tradición autoritaria que ha sufrido este país. En contraste con otros países de nuestro entorno cultural, en España todavía persiste una cierta actitud respecto a las leyes y la autoridad pública como un obstáculo potencial para el desarrollo de la libertad individual y el ejercicio de los derechos, más bien que como el resultado de la voluntad colectiva o la plataforma para el servicio de los intereses generales. Sea cual sea la naturaleza o el origen, la confianza en la administración pública y su gestión es más bien escasa en nuestro país ${ }^{43}$. Esto podría impulsar la iniciativa personal, pero parece que más bien lleva a muchos ciudadanos a no plantearse su participación en procedimientos de la administración porque no lo ven factible o posible.

Otro factor importante que explica una cierta pasividad en la vida pública es la falta de información sobre derechos y posibilidades de participación, lo que en parte es resultado de la falta de voluntad de 
legisladores y administración de dar a conocer y fomentar el uso de las posibilidades que permite la legislación (como ya ha sido indicado anteriormente).

(d) Tendencia a la politización de los debates sobre tecnología y medio ambiente. Debido a la situación política, el activismo y la concienciación sobre temas relacionados con la tecnología y el medio ambiente, resumidos en las cuatro clases de factores comentados más arriba, llegan a nuestro país con un cierto retraso con respecto a otras naciones occidentales. El desarrollo de dicha conciencia es por tanto coincidente con la transición a la democracia y con una intensa politización de la sociedad. Al mismo tiempo, no existen en España movimientos fuertes e independientes con reivindicaciones relacionadas con cuestiones ambientales o con los efectos de las tecnologías, ni tampoco partidos con representación parlamentaria que se centren en estas cuestiones (como los Verdes en algunos países europeos).

Esto, junto con la politización general de la sociedad, lleva también a una fuerte politización de las discusiones sobre medio ambiente y tecnología: muchas de estas discusiones tienden a acabar encuadradas dentro de los esquemas y debates políticos pre-establecidos. Normalmente no se convierten en debates generales sobre el tema ambiental o tecnológico, más allá de una u otra ideología específica (lo que sí sucede con más frecuencia en otros países del Norte de Europa).

Al contrario, las discusiones en España tienden a centrarse en casos específicos, y en la argumentación pesan cuestiones políticas que no tienen directamente que ver con la temática ambiental o tecnológica y que incluso pueden llegar a eclipsarla. O también se esconden polémicas políticas más generales detrás de argumentaciones ambientales.

Un ejemplo reciente: la construcción de la autovía N-III entre Valencia y Madrid, cuyo paso por la zona natural de las hoces del río Cabriel suscitó una controversia que paralizó la construcción durante años. Aquí, detrás de la polémica ambiental había otra sobre la relación entre autonomías y estado central. La discusión pública se centraba en la cuestión ambiental, pero lo decisivo fue al final la resolución de la otra controversia. Otro ejemplo de la politización es la vinculación directa a un partido político de coordinadoras contra proyectos técnicos, como por ejemplo en el caso de la "coordinadora cívica" contra un proyecto de una planta de tratamiento de residuos en Dos Aguas (Comunitat Valenciana), presidida por un concejal del partido opositor en este municipio. 
Situaciones como éstas dificultan la creación de movimientos independientes de ciudadanos sin fines partidistas y la aparición de debates centrados en las cuestiones ambientales y tecnológicas.

Otro aspecto de la politización es la integración de muchos movimientos sociales en el marco de los partidos políticos después de la transición. Las publicaciones españolas sobre participación de finales de los años 70 y principios de los 80 indican una actividad bastante amplia de movimientos independientes, no vinculados directamente a un esquema partidario, con reivindicaciones participativas, especialmente en urbanismo ${ }^{44}$. Esto es un indicio de que la situación actual de la participación en España no es simplemente el resultado de una falta de experiencia democrática a causa de los 40 años de dictadura, sino que también juega un papel la organización del sistema político español durante las dos últimas décadas.

\section{Consecuencias}

Todo lo anterior podría llevar a la conclusión de que en España no existe implicación ciudadana, lo que no es totalmente cierto. Participación pública en asuntos como, especialmente, el urbanismo existe en muchos municipios españoles y puede incluso tomar la forma de un complejo sistema organizado y permanente, como en el caso de la ciudad de Córdoba (Andalucía) ${ }^{45}$. Pero, esta participación tiene en la mayor parte de los casos unas características muy determinadas:

* Los ciudadanos como tales sólo tienden a involucrarse activamente en controversias relacionadas con la tecnología o el medio ambiente cuando éstas les afectan directa y significativamente. Se trata del llamado síndrome "No en mi patio trasero". Los ciudadanos, además, requieren entonces el apoyo organizativo y material de los grupos de interés organizados (tales como sindicatos, organizaciones ecologistas o asociaciones de consumidores). La única vía disponible suele ser entonces la resistencia social o la lenta $\dddot{y}$ costosa acción judicial.

Todo esto lo muestra una amplia gama de ejemplos actuales, como la resistencia de habitantes de los pueblos colindantes a la ampliación del aeropuerto de Barajas o a la incineradora en Valdemingómez (ambos en la Comunidad de Madrid), las manifestaciones populares en A Coruña (Galicia) en favor de un cambio en la política de residuos, o la protesta popular que paró la instalación de una planta de tratamiento de residuos tóxicos en Buñol (Comunitat Valenciana). 
Las reticencias de los ciudadanos y la politización dan con frecuencia a estas controversias unas características muy específicas: el activismo es efímero y no suele consolidarse fuera de los grupos de interés, con independencia de compromisos ideológicos o activismo político. A diferencia de lo que ocurre en muchos debates sobre proyectos tecnológicos en otros países europeos, las controversias en España raramente se vuelven debates públicos que involucren a amplias capas de la ciudadanía. En cambio, la polémica se abre paso a través de grupos organizados e incluso partidos políticos y se resuelve también dentro de este ámbito específico (otra vez sirve de ejemplo el caso de la N-III).

* La falta de debate público abierto no significa que los ciudadanos no hayan estado o estén preparados para ello. La biotecnología, por ejemplo, nunca ha sido objeto de ningún debate público en este país (sólo últimamente se dan indicios de que esta situación podría cambiar). Pero un experimento de sondeo de opinión mediante grupos de discusión llevado a cabo ya en los años 1989/90 en España demostró que ciudadanos no expertos, después de informarse sobre un asunto tan complejo como la biotecnología, eran perfectamente capaces de debatirlo en profundidad ${ }^{46}$.

* Existe también una cierta implicación ciudadana en las ONGs, que son activas a un nivel supra-regional y se preocupan por temas más amplios, aunque es todavía muy reducida en comparación con otros países europeos. Con todo, a pesar del bajo nivel de implicación ciudadana y los problemas de financiación, las ONGs han llegado a ser un factor de presión influyente. Aparte de logros como, por ejemplo, su influencia decisiva en el cambio de la política estatal de tratamiento de residuos por incineración, estos grupos representan a la opinión pública crítica porque son ellos (y no la administración) los más creíbles para la mayoría de los ciudadanos ${ }^{47}$. Así, indirectamente, se abre un tipo de participación por presión sobre los que toman las decisiones.

Lo que hay que destacar también es que mientras estos grupos presionan para ampliar las posibilidades de participación, como por ejemplo en el mencionado caso de la Ley de Biotecnología, no está claro en cambio si están dispuestos, guiados por experiencias anteriores (como la del CAMA), a dar a estas posibles vías participativas mucha importancia en la práctica ${ }^{48}$.

* A pesar de la multiplicación de controversias sobre sistemas tecnológicos a todos los niveles, la administración pública sigue manteniendo, al menos como tendencia, una concepción tecnocrática donde sigue equiparándose "técnicamente eficiente" con "políticamente adecuado». Esto lleva a la administración a buscar soluciones a la oposición 
social en la tecnificación y expertización de las cuestiones ambientales y tecnológicas. Éste ha sido, por ejemplo, el caso de la política forestal en Asturias, donde la administración intentó aplacar la oposición popular a las plantaciones de eucalipto introduciendo para ellas evaluaciones de impacto ambiental (EIA). En julio de 1990 se hizo público en Asturias el Decreto 54/90 por el que cualquier proyecto de plantación de eucalipto, en caso de sobrepasar un área mínima (entonces no especificada), debía ir acompañado de una EIA que tenía que recibir el visto bueno de la administración. Se trataba de la primera medida legislativa autonómica adoptada ante el problema social del eucalipto. El significado de su ejecución, sin embargo, fue el de transformar un problema social en una cuestión técnica durante el proceso de implementación de la ley. En este proceso, los expertos han sido los únicos encargados de la elaboración de EIAs (expertos contratados por la empresa privada) y han actuado discrecionalmente en la toma de decisiones respecto a valoración de proyectos o de denuncias (expertos de la administración). Particularmente, en la valoración de denuncias, dado que éstas se han basado mayoritariamente en la falta de EIA exigida por la ley, la respuesta de los expertos ha sido normalmente que el proyecto denunciado no sobrepasaba la unidad de área mínima (que hasta 1993 debía ser determinada en cada caso concreto) requerida para tal exigencia: la denuncia era entonces archivada ${ }^{49}$. Se trata, como han denunciado las asociaciones ecologistas involucradas en la polémica, de una aparente solución técnica para intentar silenciar protestas y permitir que todo siga igual. El resultado político ha sido inestabilidad social y ambigüedad administrativa.

Este tipo de reacción de la administración refuerza una gestión defensiva, una gestión que limita la participación a una cierta sensibilidad institucional respecto a las actitudes públicas. La participación pública se convierte entonces en una participación muy indirecta y reactiva, una forma de participación que no evita el conflicto y la resistencia social.

\section{Algunas propuestas}

Desde luego, aún más arriesgado y problemático que el diagnóstico crítico son las posibles propuestas constructivas. En cualquier caso, no se trata aquí de elaborar una receta para resolver de una vez los problemas de la participación en España, sino de formular retos y sugerencias abiertas. Proponemos la reflexión en torno a tres posibles líneas de acción: 
* En educación y formación. Un objetivo puede ser el desarrollo y consolidación de la investigación y educación sobre «ciencia, tecnología y sociedad" (CTS), del modo emprendido tímidamente por el Ministerio de Educación mediante la nueva asignatura con ese nombre que incluyen todos los bachilleratos LOGSE y los contenidos CTS que incorporan diversas asignaturas de ciencias en la nueva Educación Secundaria Obligatoria (ESO). Se trata de una innovación educativa en enseñanza media que está produciendo ya un gran crecimiento de la investigación y docencia CTS en la universidad. El propósito general no es sólo formar ciudadanos responsables y conscientes del impacto de la ciencia y la tecnología en el mundo actual, sino extender una imagen más realista y contextualizada de la ciencia y la tecnología entre los propios expertos y los responsables de la gestión pública. Obviamente, este objetivo no se limita a la educación reglada en enseñanza media y superior, sino que abarca también la formación pública a través de los medios de comunicación, museos, ciudades de la ciencia, etc.

* En la esfera legal. Otro modo de apoyar la participación pública es promover legislación y procedimientos judiciales que faciliten la litigación fundamentada por parte de ciudadanos y grupos de interés. El asesoramiento legal y técnico, a través de canales institucionales, sería entonces imprescindible para una implementación con éxito de tales medidas legislativas.

* En el ámbito político. En este contexto debemos tener en cuenta la realidad española y el cambio en la geografía de tipos de ciudadano y organización que se produce en nuestro país. Debido a la inhibición general y la politización de la participación, la participación de ciudadanos en España se da mayoritariamente en sólo dos categorías, como. ya vimos: las personas directamente afectadas y el público interesado por motivos morales o ideológicos; así como un único tipo de organización ciudadana: las ONGs que se constituyen en grupos de interés. Esta situación dificulta, por ejemplo, la participación ciudadana directa a través de audiencias o paneles. No obstante, una línea de acción factible es estimular la participación a través de grupos de interés como organizaciones ecologistas, asociaciones de consumidores, colegios profesionales, etc. De este modo, una alternativa que parece prometedora es promover mecanismos como la gestión negociada, siempre que ésta tenga al menos un carácter representativo y efectivo. Un buen procedimiento complementario, para facilitar esa representatividad, son las encuestas de opinión sobre temas conflictivos o potencialmente conflictivos en relación con la tecnología o el medio ambiente. Aunque, en este caso, sería deseable suscitar un cierto debate 
social en los medios de comunicación con el fin de prevenir un uso defensivo de tales mecanismos de sondeo. No por obvio menos importante es, asimismo, proporcionar desde la administración la información y medios materiales que hagan posible una participación activa (aun vinculada a grupos organizados) y la interacción entre ciudadanos y representantes.

\section{Conclusión}

El desafio, desde luego, no es insignificante. La conservación del medio natural, el equilibrio en el desarrollo económico, y la estabilidad democrática de los sistemas políticos, son algunas de las cosas que dependen de cómo resolvamos el reto de una gestión tecnológica adecuada. Y ésta depende cada vez más del desarrollo y la mejora de diversos cauces institucionales para una participación pública efectiva en dicha gestión, una participación que pueda reflejar mejor la voluntad de los ciudadanos y minimizar así el conflicto y la resistencia social.

Pero se trata de iniciativas que no pueden copiarse sin más de otros países donde están siendo ensayadas con éxito. Las tradiciones, los derechos y las prácticas nacionales introducen siempre unas peculiaridades que deben ser tenidas en cuenta. España no cuenta con una larga tradición participativa, y esto se manifiesta en ciertas actitudes de la población y la administración pública. Ello, sin embargo, no debería ser obstáculo para que, de acuerdo con las líneas de acción comentadas antes, traten de promoverse mecanismos participativos fundamentados en una concepción substantiva de la participación pública; por ejemplo iniciativas de participación con una efectiva con delegación de autoridad y vinculadas a grupos de interés, iniciativas donde los segmentos sociales afectados puedan ver representado su punto de vista y donde sus reclamaciones sean realmente atendidas.

Debe destacarse, finalmente, la importancia de que la participación tenga un carácter activo. Una participación reactiva identifica ésta con percepción pública o bien con mera opinión pública, entendidas como interferencia externa que es necesario incorporar a la gestión (con lo cual serían suficientes mecanismos de sondeo o, a lo sumo, consultivos). Entender de este modo la participación pública es crear riesgos de manipulación e inestabilidad, así como omitir una aportación potencialmente valiosa (la del conocimiento popular local y los actores sociales implicados) en la resolución de problemas relacionados con tecnología ${ }^{50}$. La complejidad de los problemas abordados actualmente 


\section{Participación pública en política tecnológica}

por la ciencia y la tecnología, y la presencia de valores e intereses "externos" en el conocimiento especializado, hacen de la pluralidad de perspectivas y la participación social un bien valioso tanto desde un punto de vista político como desde el estrictamente práctico.

De este modo, en nuestra opinión, también en nuestro país pueden promoverse cauces institucionales que faciliten unas políticas tecnológicas más eficaces; así como programas para mejorar el conocimiento y la concienciación de las personas que son necesarios para un enriquecedor aprendizaje social y una democratización en profundidad de la gestión pública en innovación tecnológica e intervención ambiental. España es ciertamente diferente, y la tecnología no es un asunto sencillo, depende de nosotros ver en ello un obstáculo desalentador o bien un desafío aún más estimulante.

\section{Agradecimientos}

Queremos expresar nuestro agradecimiento a Marta González y José Luis Luján por su discusión crítica y colaboración desinteresada para mejorar diversas partes del texto. Por sus comentarios a una versión anterior de este trabajo, también estamos en deuda con los profesores Imre Hronszky, Tihamer Margitay y Josephine Stein. Los posible errores contenidos en la versión final son, por supuesto, de nuestra exclusiva responsabilidad. El apoyo material del Ministerio de Educación (DGICYT, PS92/0121) y el Vicerrectorado de Investigación de la Universidad de Oviedo ha hecho posible la realización de este trabajo.

\section{Notas}

1 En rigor deberíamos hablar de cambio científico-tecnológico y ambiental, así como de políticas científico-tecnológicas y ambientales. Para abreviar, utilizaremos con frecuencia las expresiones "cambio tecnológico" y «política tecnológica». No obstante, debemos recordar que la tecnología tiene un crucial componente científico y que, habitualmente, los programas de intervención ambiental suponen conocimiento especializado y la aplicación de tecnologías materiales u organizativas. Véase, en general, González García et al. (1996).

2 El esquema que sigue esta sección es una adaptación del diagnóstico que puede encontrarse en Nelkin (1984).

${ }^{3}$ Sobre estudios sociales, véase, por ejemplo, Jasanoff et al. (1995) y González García et al. (1996). Dos textos, en esta corriente, escritos claramente para el gran público son Barnes (1985) y Collins y Pinch (1993). 


\section{José A. Cerezo, José A. Méndez Sanz y Oliver Todt}

4 Véase la extensa discusión del tema en Stehr (1994: 166 ss.).

5 Como muestra de la primera clase de estudios, véase Wynne (1992); respecto a la segunda, véase Luján y Moreno (1993). Véase, en general, Wynne (1995).

6 Véase Beck (1986).

7 Un panorama general sobre la diversidad actual de estudios sobre la recepción de la ciencia por parte del público, los llamados estudios de «public understanding of science», puede encontrarse en Wynne (1995).

8 Se trata de los tres argumentos que desarrolla y populariza Daniel Fiorino, de la Environmental Protection Agency norteamericana, en (1990: 227-228). Aunque Fiorino presenta los argumentos con respecto a situaciones de riesgo ambiental, es razonable que puedan extenderse a la participación pública en política científico-tecnológica o, de forma más amplia, a la participación pública en política en general.

9 Véase Elliott y Elliott (1976) como una alerta temprana que, basada en la tradición de pensamiento marxista, anuncia un cambio general de sensibilidad.

10 Véase Nelkin (1984: 25), cuya clasificación general es también adaptada en la presente sección.

11 Véase una fuerte crítica a este compromiso de los científicos en Handler (1980: 95), donde se defiende una estricta separación entre los roles profesional y ciudadano en el científico y se califica de anomía la participación de científicos en el movimiento ambiental y de consumidores.

12 Sobre organizaciones obreras y de consumidores, puede consultarse Elliott y Elliott (1976: 215 ss.)

13 Véase Rose y Rose (1976).

14 Véanse Fiorino (1980: 229-230), Laird (1993: 353-355), Krimsky (1984:45), Rip (1986).

15 Véase Rip (1986) para una argumentación a favor de la participación activa donde se destaca la importancia del disenso y las controversias para el aprendizaje social y la anticipación de impactos en el desarrollo tecnológico.

16 Estos requisitos derivan, como es obvio, de una determinada teoría general de la democracia. Aunque no es éste el lugar para entrar en la discusión de las distintas concepciones de la democracia, sí resulta relevante señalar que, en la literatura actual sobre participación pública, el debate gira en torno a dos grandes teorías: la de la participación directa y la pluralista (o poliarquía, liberalismo de grupos de interés, etc.). La diferencia básica entre ambas estriba en que los pluralistas entienden por democracia la libre competencia entre distintos grupos de interés voluntariamente organizados y por participación la búsqueda de resultados consensuados concretos en una sociedad ya democráticamente constituida, mientras que, por su parte, los defensores de la participación directa entienden que es el individuo en cuanto individuo quien debe participar e incidir en la gestión pública, interesando aquí no tanto el resultado como el proceso de participación que hace de él un ciudadano y ayuda a construir la democracia. De esta diferencia básica deriva una diferencia a la hora de evaluar el grado de cumplimiento de los requisitos que hacen democrática una forma de participación pública, requisitos en los que, sin embargo, básicamente coinciden: amplia y significativa participación ciudadana (véase Laird, 1993: 343-349). Las condiciones anteriores recogen en cierto modo esos requisitos comunes. Con respecto a la teoría de la participación directa, véase, por ejemplo, Fiorino (1990). Para la teoría pluralista, puede consultarse, por ejemplo, Dahl (1989). Una comparación 


\section{Participación pública en política tecnológica}

entre ambas, respecto a los tipos de mecanismos participativos favorecidos, puede encontrarse en Laird (1993).

17 Véanse Krimsky (1984: 45 ss.), Fiorino (1990: 230 ss.), Laird (1993: 350 ss.). Con todo, es muy interesante la información que brinda Villasante (1995: 55-90) sobre participación pública en Latinoamérica, una zona que no se suele señalar como referente pero a la que habrá que ir prestando cada vez mayor atención.

18 La legislación española contempla la exposición pública de evaluaciones de impacto ambiental y otro tipo de informes relacionados con la política tecnológica y ambiental. Sin embargo, la información insuficiente y la falta de asesoramiento dificultan en gran medida el acceso de los ciudadanos a los informes técnicos. Una discusión más detallada se encuentra, más adelante, en el texto principal. Para las audiencias públicas realizadas por organismos como la Environmental Protection Agency en Estados Unidos, véase Fiorino (1990).

19 Véanse, por ejemplo, el programa de gestión participativa desarrollado al norte del Estado de Victoria (Australia) para resolver el problema de la salinidad de suelo y aguas, en Syme y Eaton (1989).

20 Entre los modelos teóricos, destacan la propuesta de K. Shrader-Frechette (1985a y 1985b) y el modelo de T. Burns y R. Ueberhorst (1988: secc. 4.3). Para los modelos realizados, véase Crosby et al. (1986). Entre éstos destaca, en el ámbito evaluativo, el de la NOTA (Organización Neerlandesa de Evaluación de Tecnologías): véanse Boxsel (1994), y Rip y van den Belt (1988).

21 Con carácter decisorio, el Cambridge Experimental Review Board pudo regular la investigación sobre el ADN recombinante en la ciudad de Cambridge (Massachusetts): véase Laird (1993: 352). Como ejemplo de panel consultivo no decisorio, véase el caso de la estrategia regional de Kiwanaw (para combinar protección y desarrollo industrial al sur de Perth, Australia) en Syme y Eaton (1989).

22 Un mecanismo de este tipo se está ensayando por ejemplo en Alemania: las células de planeamiento. Formadas por 25 ciudadanos comunes liberados de sus quehaceres por un tiempo determinado, han de elaborar una opinión sobre un proyecto determinado. Pueden informarse, discutir y hacer propuestas. Véase Villasante (1995: 285).

${ }^{23}$ Las encuestas son un mecanismo que, en Europa, ha sido especialmente promovido por la NOTA. Véanse Boxsel (1994) y Schot (1992).

24 Véase Nelkin (1984: 30). Fuera de los ámbitos mencionados, una forma de participación que debe también señalarse es el consumo en una economía de mercado. Sean frigoríficos, alimentos o prendas de vestir, el consumo diferencial de productos tecnológicos permite ejercer una forma de control Social sobre los procesos de innovación. Por supuesto, este control sólo es posible en países cuyas legislaciones al respecto obliguen al etiquetado detallado de productos. Véase Todot y Luján (1997).

25 Para su evaluación detallada desde las dos grandes teorías actuales de la democracia, véanse Fiorino (1990: 236-238) y Laird (1993: 343-350).

${ }^{26}$ Véase Fiorino (1988) y Laird (1993).

27 Aunque, desde luego, las principales teorías comparten lugares comunes (los lugares que hemos trata de recoger mediante los criterios formulados en el texto principal).

28 Véase Syme y Eaton (1989).

29 Véase Wynne (1992), y López Cerezo y González García (1997).

$30 \mathrm{El}$ ejemplo se encuentra en Wynne (1989), véanse otros casos análogos en Wynne (1989) y (1992). 
31 Ley 38/95, BOE no. 297: 13-12-1995.

32 Una primera valoración de la Ley de Información Ambiental y de su funcionamiento en la práctica desde el punto de vista de una ONG se encuentra en Herranz et al. (1996).

33 Sobre la Ley de Biotecnología (Ley 15/84, BOE no. 133: 4-6-1994), véase Borillo (1994) y Luján et al. (1996).

34 Según comunicación personal de Elisa Barahona (Ministerio de Medio Ambiente) del 11-6-1996.

35 Sobre legislación en urbanismo y participación, véase Enguita Puebla (1985).

36 Con el ingreso de España en la Unión Europea en 1986, la legislación española tuvo que hacerse eco de la Directriz Comunitaria 85/377/CEE de junio de 1985 sobre Evaluación de Impacto Ambiental; introducida en nuestro país por el Real Decreto Legislativo 1302/86 y su reglamento aprobado por Real Decreto 1131/88.

37 Sobre la participación ciudadana en el funcionamiento de la administración española, véase Castellà (1992).

38 Según comunicación personal de Jesús Cabasés (AEDENAT) del 20-11-1996.

39 Véase Herranz et al. (1996).

40 Véanse López Cerezo y González García (1993) y (1997).

41 Sobre la relación entre desarrollo del Estado de Bienestar y la sociedad civil en España, véase por ejemplo Cortina (1994).

42 Véase Luján y Moreno (1993).

43 Como lo ponen de manifiesto las opiniones sobre el papel deseado de la administración en la regulación de la biotecnología en Moreno et al. (1992), pero también encuestas: Luján y Moreno (1993).

44 Para algunos ejemplos, véase la sección especial sobre «participación popular en el planeamiento urbano* (CEUMT, no. 10, 1979) y Enguita Puebla (1985).

45 Véase Villasante y Alberich (1993).

46 Véase Moreno et al. (1992).

47 Véase Luján y Moreno (1993).

48 Esto se desprende de comunicaciones personales de representantes de varias ONG, entre ellos Isabel Bermejo del Fondo Patrimonio Natural Europeo y Jesús Cabasés de AEDENAT, de noviembre de 1996.

49 El área mínima de cultivo agrícola y forestal sólo se determina en el Decreto 84/92 de 30 de diciembre. Desde entonces, será la falta estimada de impactos ambientales significativos (de sincidencia en el medio naturalw) lo que continúe justificando en muchos casos la carencia de EIAs, o bien de su falta de exposición pública. Véase López Cerezo y González García (1993).

50 Véase, por ejemplo, Fiorino (1990) y Wynne (1992).

\section{Bibliografía}

BARNES, B. (1985): Sobre ciencia. Labor, Barcelona, 1987.

BECK, U. (1986): Risk Society, Sage, Londres, 1992.

BORRILLO, D. (1994): Spanish Law on Genetically Modified Organisms, IESA documento de trabajo 94-14. IESA/CSIC, Madrid. 


\section{Participación pública en política tecnológica}

BoxSEL, J. van (1994): «Constructive Technology Assessment: A New Approach for Technology Assessment Developed in the Netherlands and its Significance for Technology Policy», en: G. Aichholzer y G. Schienstock (eds.): Technology Policy: Towards an Integration of Social and Ecological Concerns. De Gruyter, Berlín-Nueva York, 1994.

Burns, T. R. y Ueberhorst, R. (1988): Creative Democracy: Systematic Conflict Resolution and Policymaking in a World of High Science and Technology. Praeger, Nueva York.

CASTELLA, J. (1992): «La participación ciudadana en la administración pública», Revista Vasca de Administración Pública 34: 39-83.

Coluins, H. y PINCH, T. (1993): The Golem: What Everyone Should Know About Science. Cambridge University Press (trad. cast. en Critica: El gólem), Cambridge.

Cortina, A. (1994): La ética de la sociedad civil. Anaya, Madrid.

Crosby, N., Kelly, J. M. y Schaefer, P. (1986): «Citizens Reviews Panels: A New Approach to Citizen Participation*, Public Administration Review 46: 170-178.

DAHL, R. (1989): Democracy and its Critics. Yale University Press, New Haven.

Elliot, D. y ElliotT, R. (1976): El control popular de la tecnología. Gustavo Gili, Barcelona, 1980.

Enguita Puebla, A. (1985): "La participación ciudadana en el urbanismo». Revista de Derecho Urbanístico 91:63-80.

FIoRINO, D. J. (1990): «Citizen Participation and Environmental Risk: A Survey of Institutional Mechanisms». Science, Technology, and Human Values 15/2: 226-243.

GonzÁlez Garcta, M. I., LOPEZ CereZo, J. A. y LuJÁN, J. L. (1996): Ciencia, tecnología y sociedad: una introducción al estudio social de la ciencia y la tecnología. Tecnos, Madrid.

Handler, P. (1980): «Public Doubts about Science», en: R. Chalk, Science, Technology, and Society: Emerging Relationships. American Association for the Advancement of Science. Washington D.C., 1988.

HERRANZ, D.L. et al. (1996): El derecho de acceso a la informacion sobre medio ambiente. AEDENAT, Madrid.

JASANOFF, S. et al. (eds.) (1995): Handbook of Science and Technology Studies. Sage, Londres.

KRIMSKY, S. (1984): «Beyond Technocracy: New Routes for Citizen Involvement in Sociàl Risk Assessment;, en: Petersen (1984).

LAIRD, F.N. (1993): «Participatory Analysis, Democracy, and Technological Decision Making*. Science, Technology, and Human Values 18/3: 341-361.

LOPEZ CEREZO, J.A. y GoNZALEZ GARCfA, M. I. (1993): «The Many Faces of Uncertainty: Forestry Research and Policy in Northern Spain», en: D. Procházková (ed.), Environmental Monitoring and Adjacent Problems. Czech Ecological Institute and Ministry of Environment Press, Praga, 1993.

LOPEZ CEREZo, J.A. y GonZAlez Garcfa, M. I. (1997): «Lay Knowledge and Public Participation in Technology and Environmental Policy», en: C. Mitcham (ed.), Research in Philosophy and Technology, Vol. 16, pp. 33-48. JAI Press, Greenwich (CT).

LUJÁN, J. L. y MORENO, L. (1993): «La percepción pública de la biotecnología en España», contribución a II Jornadas de Investigación sobre las Relaciones entre Ciencia, Tecnología, Economía y Sociedad. Universidad de Salamanca, Grupo EPOC, 1993. 


\section{José A. Cerezo, José A. Méndez Sanz y Oliver Todt}

LUJAN, J.L. et al. (1996): «Spain: Transposing EC Biotecnology Directives through Negotiation. Science and Public Policy 23/3: 181-184.

MORENO, L. et al. (1992): Biotecnología y sociedad. MOPT, Madrid.

NelkIN, D. (1984): «Science and Technology Policy and the Democratic Process", en: Petersen (1984).

Petersen, J. C. (ed.) (1984): Citizen Participation in Science Policy. University of Massachusetts Press, Amherst.

RIP, A. (1986): «Controversies as Informal Technology Assessment». Knowledge: Creation, Diffusion, Utilization 8/2: 349-371.

RIP, A. y VAN DEN BELT, H. (1988): «Constructive Technology Assessment: Toward a Theory*, documento de trabajo, Universidad de Amsterdam.

Rose, H. y Rose, S. (1976): La radicalización de la ciencia. Nueva Imagen, México, 1980.

ScHOT, J. W. (1992): «Constructive Technology Assessment and Technology Dynamics: The Case of Clean Technologiesw. Science, Technology, and Human Values 17/1: 36-56.

Shrader-Frechette, K. (1985a): Science Policy, Ethics, and Economic Methodology. Reidel, Dordrecht.

SHRADER-FrECHETTE, K. (1985b): «Technology Assessment, Expert Disagreement, and Democratic Procedures", en: Research in Philosophy and Technology, Vol. 8, Nueva York, JAI Press, 1985.

STEHR, N. (1994): Knowledge Societies. Sage, Londres.

SYME, G. J. y Eaton, E. (1989): «Public Involvement as a Negotiation Process». Journal of Social Issues 45/1: 87-107.

TODT, O. y LUJAN, J. L. (1977): "Labelling of Novel Foods, and Public Debate». Science and Public Policy 24/5: 319-326.

VIllaSANTE, T. (1995): Las democracias participativas. De la participación ciudadana a las alternativas de sociedad. HOAC. Madrid.

VillasANTE, T. y ALBERICH, T. (1993): «Experiencia de participación ciudadana en municipios». ALFOZ n. $.^{\circ} 104 / 105$.

WYNNE, B. (1989): «Frameworks of Rationality in Risk Management: Towards the Testing of Näve Sociology», en: J. Brown (ed.), Environmentl Threats. Analysis, Perception, Management. Random, Belhaven, 1989.

WYNNE, B. (1992): «Uncertainty and Environmental Learning». Global Environmental Change 2: 111-127.

WYNNE, B. (1995): «Public Understanding of Science», en: Jasanoff et al. (1995). 